\title{
Efficacy of rowing, backward wheeling and isolated scapular retractor exercise as remedial strength activities for wheelchair users: application of electromyography
}

\author{
LM Olenik, JJ Laskin, R Burnham, GD Wheeler and RD Steadward \\ The Rick Hansen Centre, WI-67 Van Vliet Complex, University of Alberta, Edmonton, Alberta, T6G 2H9, \\ Canada
}

\begin{abstract}
Shoulder dysfunction due to regular wheelchair use is a common problem among people with spinal cord injuries. As a remedial measure, strengthening of the scapular retractor muscles has been suggested. Electromyographical analysis was utilized to examine scapular retraction muscle use during rowing, backward wheeling and a standardized scapular retraction exercise in seven people with spinal cord injuries and seven able bodied subjects. In addition, a pilot study using indwelling electrodes was completed to validate the use and placement of surface electrodes. Both rowing and the standardized scapular retraction exercise recruited higher levels of retractor involvement than backward wheeling. We suggest that rowing, because of its value as a cardiovascular exercise and high level of retractor recruitment, is an appropriate and effective means of remediating scapular retractor weakness.
\end{abstract}

Keywords: scapular retractor muscle; electromyography; wheelchair users; remedial strength activity

\section{Introduction}

Adapted physical activity literature suggests that people who use manual wheelchairs have a higher incidence of shoulder pain and dysfunction than their able bodied counterparts. ${ }^{1-5}$ Various dysfunctions have been isolated and identified, most commonly rotator cuff impingement syndrome, ${ }^{1}$ but also subacromial bursitis, ${ }^{3}$ and general muscle soreness. ${ }^{2,4,5}$

Many factors may contribute to the high prevalence of rotator cuff impingement syndrome found among the wheelchair dependent including overuse, repetitive overhead and internal rotation impingement positioning, as well as axial weight bearing through the shoulder. ${ }^{6}$ As suggested by a recent study, an additional factor may be muscle imbalance around the shoulder (weakness of the rotators in relation to the deltoid). The internal and external rotator muscles of the rotator cuff (subscapularis, infraspinatus and teres minor) help to control cephalad migration of the humeral head caused by the upward pull from deltoid contraction and upward thrust from axial loading of the arm during wheelchair propulsion. Insufficient humeral head control results in acromiohumeral space encroachment and impingement of the rotator cuff and subacromial bursa. ${ }^{7}$ As the shoulder rotators originate from the scapula, it is imperative that the scapular stabilizing muscles, which counterbalance the pull of the shoulder rotators, be competent. The scapular retractors (rhomboids and mid-trapezii) fulfil this function. Excessive stretching or weakness of the scapular retractors allow the scapula to assume an excessively protracted position. It has been demonstrated that the width and configuration of the subacromial space is significantly less in a scapular protracted position as compared to one of scapular retraction. ${ }^{7}$ In able bodied individuals with a rotator cuff impingement syndrome, excessive range of the scapular retractors, as measured by increased lateral scapular glide, has been documented. ${ }^{8}$ Excessive scapular protraction would disturb the normal length-tension relationship of the shoulder rotators muscles and reduce their efficiency in controlling the upward migration of the humeral head into the acromiohumeral space. ${ }^{9}$ Therefore, successful strengthening of the shoulder rotators muscles requires adequate scapular stabilization, particularly that provided by the scapular retractor muscles.

In addition, similar shoulder dysfunctions have been described in young wheelchair athletes with the suggestion that chronic or arthritic processes could develop if preventive measures are not taken. ${ }^{10}$ However, convenient and effective retractor strengthening exercises have not yet been described for people who use wheelchairs.

Electromyographical (EMG) analysis can be used to determine the efficacy and timing of muscle recruitment with exercise.

The primary purpose of this study was to explore the efficacy of various exercises in recruiting scapular retractor muscles. In doing so we recommend a form of training to remediate, and potentially prevent, the suggested posterior/anterior muscle imbalance. ${ }^{1}$ 


\section{Subjects and methods}

Seven subjects with spinal cord injuries (C4-7) and seven able bodied subjects gave informed consent to participate in this study after approval was granted by an institutional ethical review committee. All subjects were volunteers, over 18 years of age, and recreationally active. Any subject with shoulder pathology which would place him or her at risk or jeopardize the results of the study was excluded.

Each subject completed three $20 \mathrm{~s}$ trials consisting of stationary rowing, backward wheeling on rollers, and standardized scapular retraction (SSR) in a prone position. Each of these exercises was reported three times for a total of nine trials.

Subjects completed the rowing protocol from a seated, stationary position approximately $1 \mathrm{~m}$ from a wall mounted Biokinetics pulley system which allowed for (frictional) resistance only during retraction. The retraction phase began with arms extended and comprised a pull to the mid-sternum (Figure 1a, b). When necessary, trunk stabilization was provided. Backward wheeling was completed on a set of double wheelchair rollers and included three intermittent pulls every $10 \mathrm{~s}$, that is, six pulls per trial. The standardized scapular retraction required a prone position on a height
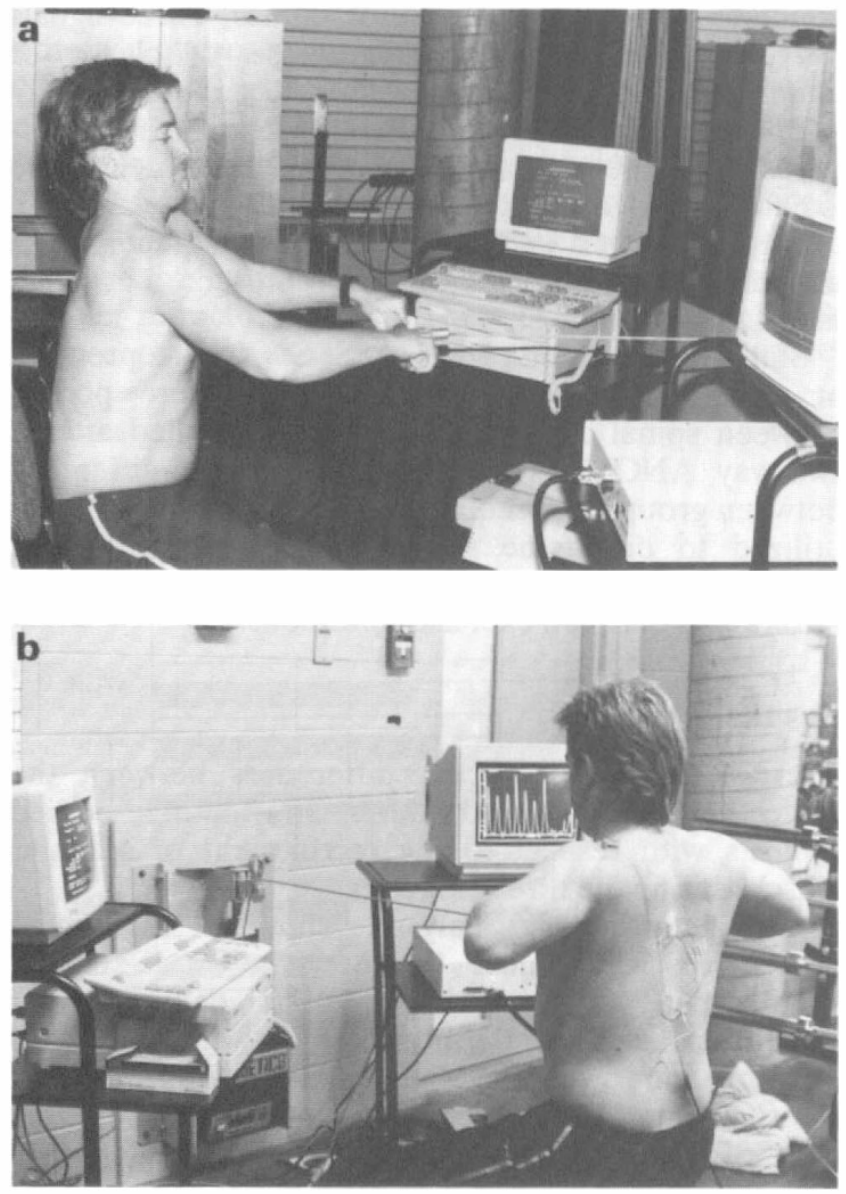

Figure 1 (a) Photograph of extended row stroke. (b) Photograph of row stroke, standardized to mid-sternum adjustable weight bench with the left arm hanging freely over the edge, elbow extended. The subject was asked to produce a movement which moved the scapula medially (Figure 2). This movement was unilateral, unlike the other two exercises; however muscle recruitment was measured unilaterally in all three protocols.

When recording the EMG signals the subjects were told to work at a comfortable pace and effort. The mechanical resistance was set at level zero and resistance due to gravity, friction, and inertia was implicit in each of the exercises. Therefore, for the purposes of analysis, the workload for each of these exercises was considered to be effectively zero.

In order to record the EMG signals of the muscles, or portions of muscles, responsible for scapular retraction, surface electrodes were placed unilaterally in a standardized position midway between the medial border of the scapula and the fourth-sixth spines of the thoracic vertebrae (Figure 3). ${ }^{11,12}$ Therefore, recordings were being made from the rhomboids, and mid-trapezius muscles. ${ }^{13,14}$ Placement 'validity' was determined by two manual muscle tests known to isolate scapular retractor muscles. ${ }^{15}$ The electrodes were not moved between the different test exercises nor during the test runs. Recordings from the elec-

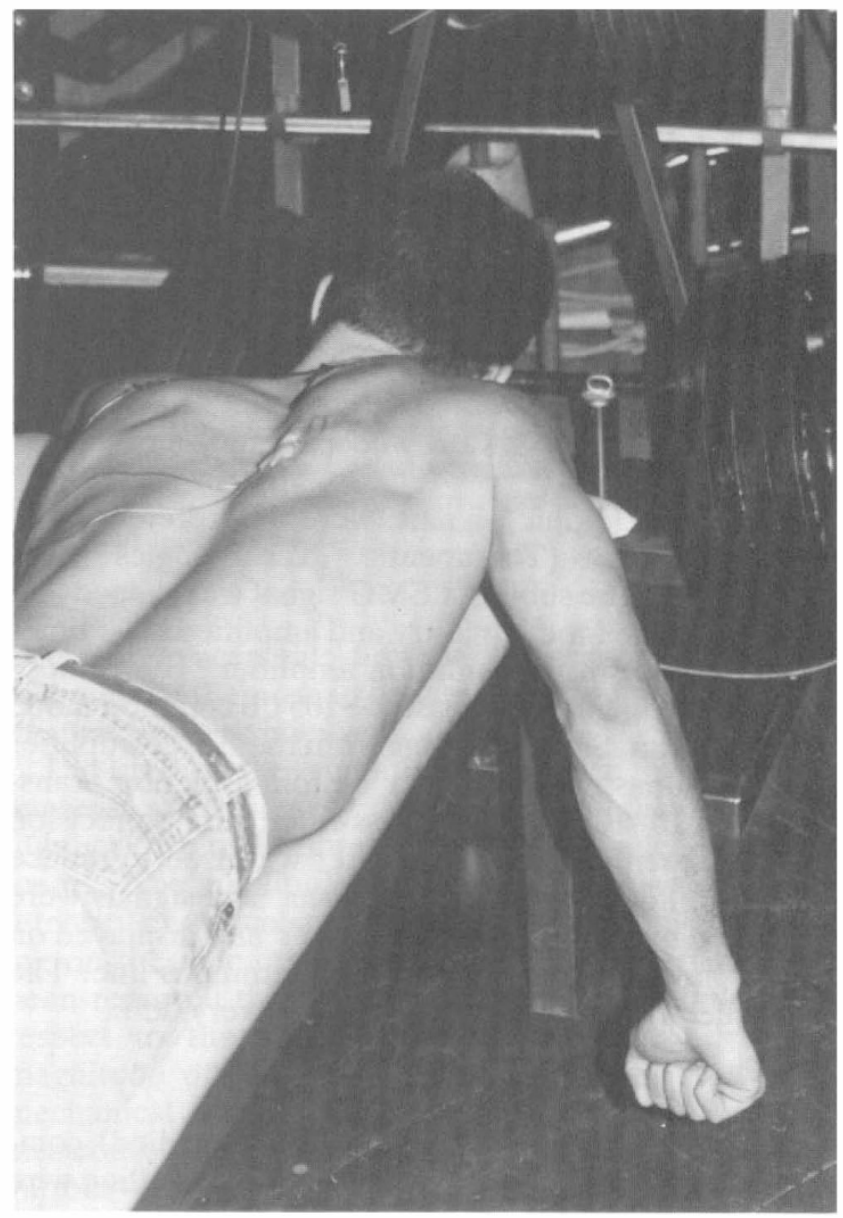

Figure 2 Photograph of the standardized scapular retraction movement 


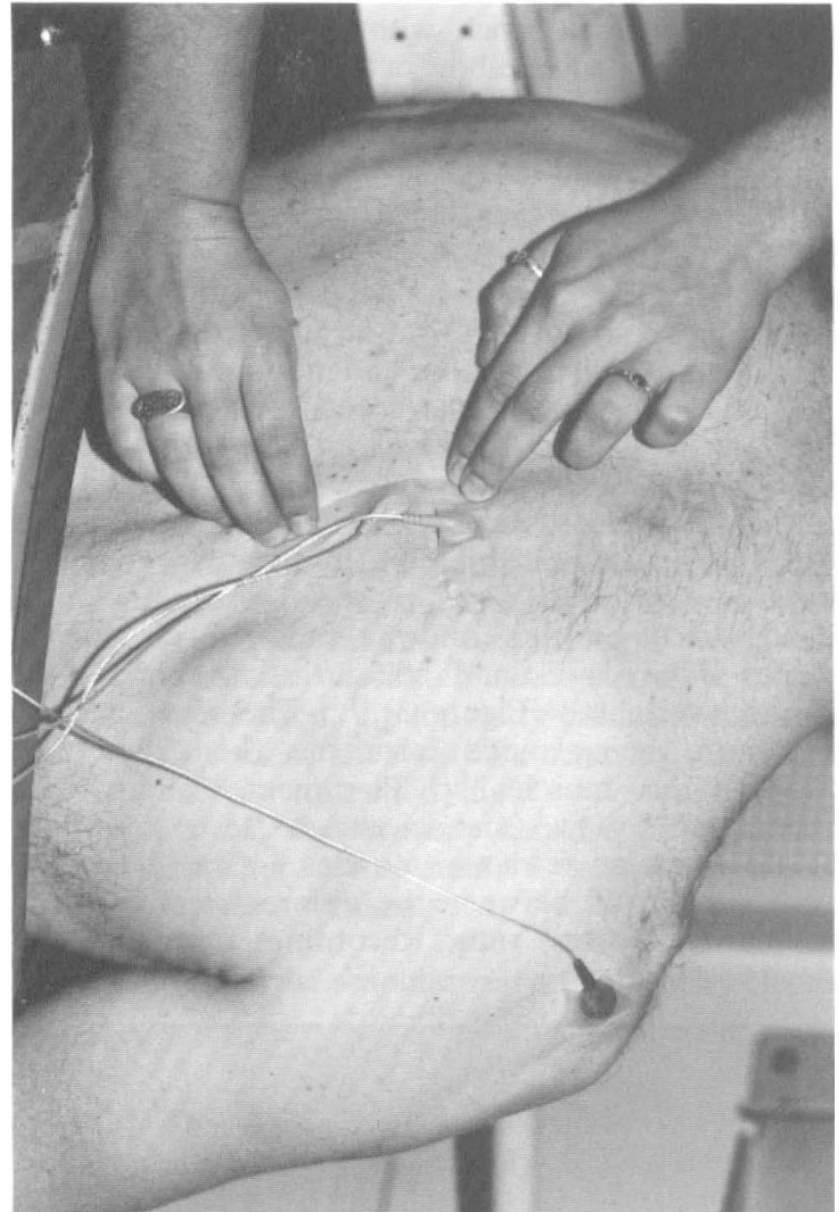

Figure 3 Photograph of the electrode placement on subject's back

trodes were used to compare amplitudes and turns between rowing, backward wheeling and the scapular retraction exercise. ${ }^{12,16,17}$

Electromyographic signals were interpreted by a NeuroEducator (Therapeutic Technologies Inc, Tampa, FL). The subject's EMG signal was shaped and clamped by the input circuitry and amplified 8000 times by a low noise instrumentation amplifier. The components had to maintain at least a $-105 \mathrm{db}$ common mode rejection ratio. Signals coming from the EMG amplifier were then passed through a custom isolation transformer, a $10-1000 \mathrm{~Hz}$ bandpass filter, and a precision full wave rectifier. A cardiac filter was used to reduce the possibility of signal interference. The signals were integrated over $0.1 \mathrm{~s}$, calibrated in $\mathrm{uV}$ and displayed on a color monitor in the form of a continuous line. The data collected were then averaged for every $0.5 \mathrm{~s}$.

\section{Pilot test}

Four of the fourteen subjects (all able bodied) completed the above test protocol using an indwelling wire electrode in conjunction with the surface electrodes and, on a separate occasion, a needle electrode with surface electrodes. Recordings from these indwelling electrodes were used to check for synchronization between motor unit signals from the indwelling and surface electrodes. This comparison was made in order to validate the use and anatomical placement of the surface electrodes. ${ }^{17}$

Wire electrodes consisted of an indwelling platinum wire placed with an insertion cannula approximately $0.45 \times 29 \mathrm{~mm}$.

Needle electrodes consisted of a stainless steel needle coated with 50 um teflon layer in such a manner that only the tip was left uninsulated. Both the needle and wire cannula were inserted through the middle trapezius and rhomboid muscles until the posterior aspect of the fifth or sixth thoracic rib was contacted. The cannula was then withdrawn leaving the wire embedded in the rhomboid muscle and approximately $1 \mathrm{~mm}$ dorsal to the rib. ${ }^{11,12}$

Because of the discomfort and risk of breakage or infection inherent in the use of indwelling electrodes in dynamic assessment, the researchers determined that the four subjects with 10 trials each were sufficient to demonstrate the placement validity of using surface electrodes.

\section{Data analysis}

For each subject nine $20 \mathrm{~s}$ runs (recorded by the surface electrodes) were analyzed per exercise. Each run contained at least five amplitude signals. Three signals per run were chosen on the basis of clarity of amplitude. Therefore, for each subject there were 27 segments analyzed for each test exercise. The amplitudes of the retraction phase of the movement were isolated and averaged. The total EMG data were then averaged for every subject per exercise and recorded as a mean, that is, each subject was represented by three mean exercise values.

Statistics were completed on SPSS-X IBM program at the University of Alberta. Data were pooled between spinal cord injured and able bodied after a two-way ANOVA revealed no significant difference between groups. Subsequently a one-way ANOVA was utilized to determine significance of the exercises. Post-hoc analysis (Tukey) was computed to determine differences between exercise conditions.

\section{Results}

There were no significant differences between the subjects with spinal cord injuries and the able bodied subjects with respect to the recruitment of scapular muscle during the three exercises.

The statistical analysis showed significant $(P=0.007)$ differences between the three testing conditions. Post-hoc Tukey analysis identified that there were significant differences between backward wheeling and rowing; and backward wheeling and SSR. No significant difference was found between rowing and SSR. Rowing and SSR consistently elicited considerably higher EMG amplitudes when compared to backward wheeling. The data suggest that the muscles used in scapular retraction are preferentially recruited 
in both rowing and SSR exercises (Figure 4). This is consistent with literature pertaining to scapular muscle rehabilitation in able bodied athletes. ${ }^{14}$

With respect to the pilot study results, a visual analysis ${ }^{16}$ was completed on the data collected on the four subjects who participated in the wire/needle electrode portion of the study. Turns and amplitudes were quantified by the NeuroEducator and independently analyzed by the investigators according to methods discussed by Gilai. ${ }^{17}$ The retraction phase segments were found to be within $0.5 \mathrm{~s}$ for both the indwelling and surface EMG signals (Figure 5). It was therefore determined that the surface electrodes alone were measuring signals produced by the muscles responsible for scapular retraction.

\section{Discussion}

It is evident that shoulder problems are prevalent among wheelchair users. ${ }^{1-5,10}$ Shoulder muscle imbalance of wheelchair athletes, associated with rotator cuff impingement syndrome have been documented. The relative weakness of the shoulder rotators cannot be rehabilitated until the strength and coordination of the scapular retractors is adequate to provide scapular stability. ${ }^{2-5}$ Therefore, there is a need for appropriate methods to strengthen the scapular retractors of people who use wheelchairs. ${ }^{10,18}$ For both remedial and preventative purposes, rowing, backward wheeling, and SSR exercises were investigated. This study found rowing and SSR to be more effective in activating scapular retractors than backward wheeling.

The initial use of indwelling electrodes placed directly into the rhomboid trapezius muscles provided evidence that surface electrodes were reliably detecting scapular retractor muscles. These investigators support the use of indwelling electrodes to validate surface

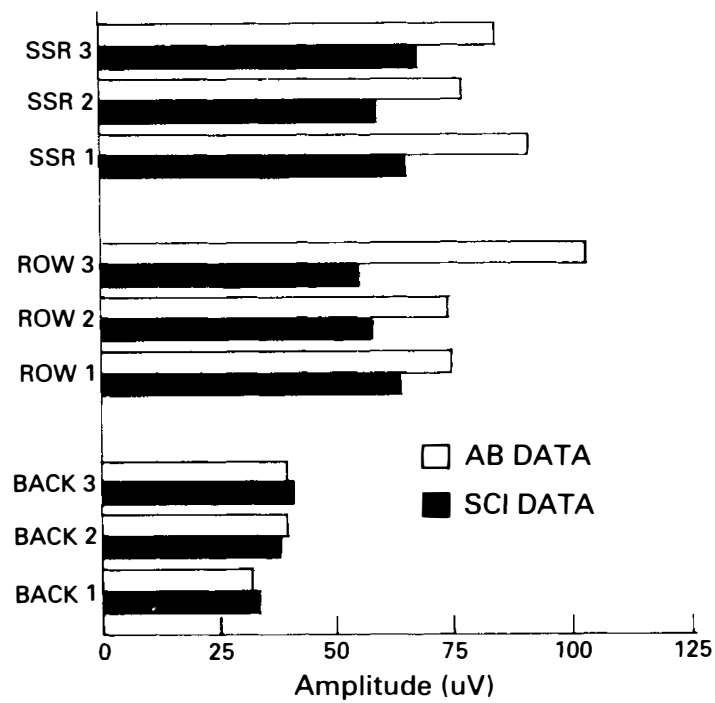

Figure 4 Graph demonstrating the differences between the three test exercises in terms of EMG amplitudes. Able bodied and spinal cord injured data is shown separately to illustrate the analogous trend between the two groups
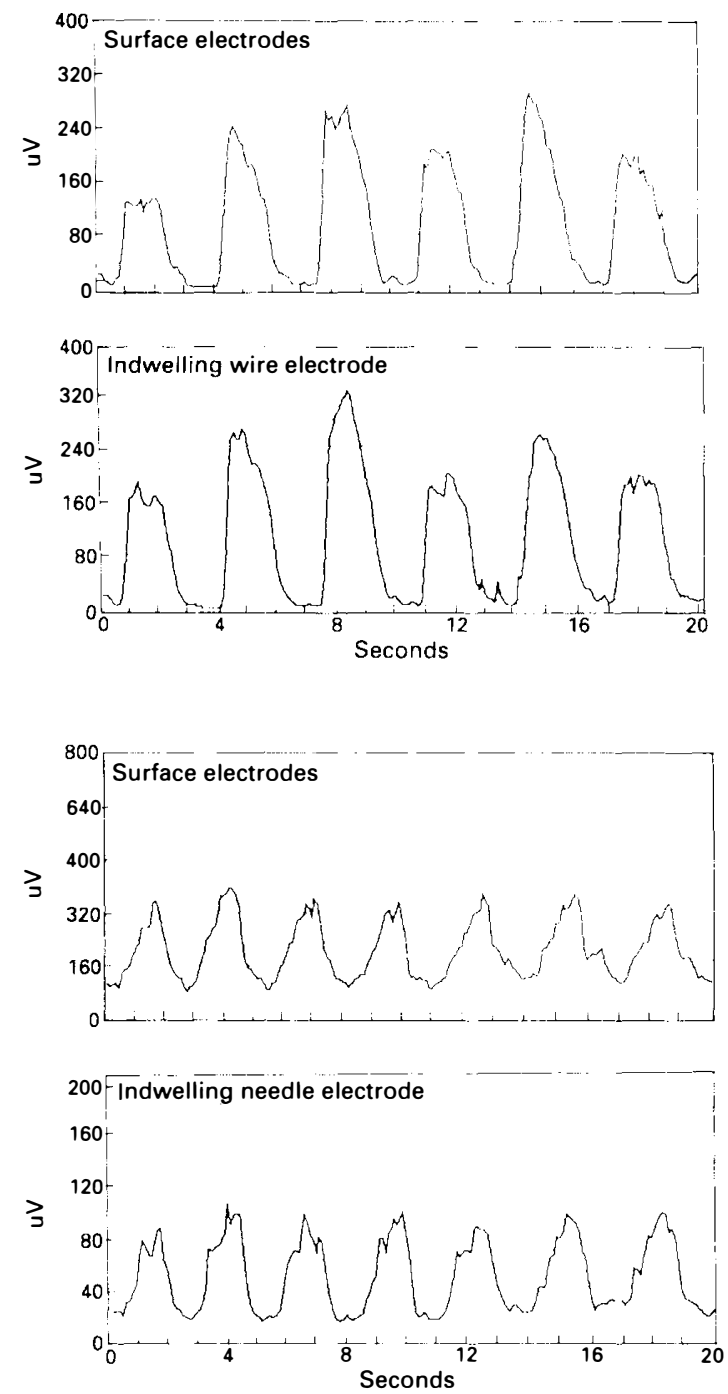

Figure 5 Graph showing electrode placement validity: surface to needle, and surface to wire. Note that the validity is with regard to synchronous turns and not necessarily with equivalent amplitudes

electrode placement; however, they are hesitant to recommend their use in dynamic assessment because of the pain and risk involved.

The size of the amplitudes recorded by an EMG analysis are primarily a function of the type and number of muscle fibres recruited by a specific movement. ${ }^{17}$ The amplitude should give an indication of the intensity of muscular effort, which may change depending on the amount of resistance provided. It has also been reported that the position of the electrodes with respect to the zone of innervation may affect the magnitude of the signal. ${ }^{19}$ In the present study, the mechanical workload was effectively zero and the electrodes were not moved or removed between the various testing conditions; therefore it seems that a valid comparison between the three exercises can be made.

Backward wheeling, though producing some degree 
of scapular retraction activity, is not as effective in retractor recruitment as rowing and SSR. Furthermore, this study found backward wheeling to be mechanically awkward on conventional wheelchair rollers. Many athletes, however, practice backward wheeling on a track in order to strengthen posterior muscles. In fact, one subject who produced high backward wheeling amplitudes, incorporated backward wheeling into her regular fitness training program.

The cardiovascular advantages of using rowing ${ }^{20}$ as a remedial strength exercise for retractor muscles reinforces the value associated with this form of training. For people with spinal cord injuries this form of training may be particularly beneficial with the inclusion of a functional electrical stimulation component to assist lower limb movement as indicated in a previous study by these investigators. ${ }^{20}$ Although the SSR showed the highest amplitudes, suggestive of more muscle recruitment, this movement has little cardiovascular benefit and requires strict adherence to proper technique.

In conclusion, these findings, along with consistent documentation on the able bodied population, ${ }^{21}$ support the validity of rowing and SSR as effective training tools and possible rehabilitative treatments. ${ }^{14,18}$ As the goal of this study was to identify possible remedial exercises for shoulder dysfunction, further investigation on the long term effects of such a training program is warranted.

\section{Acknowledgements}

The authors would like to extend their gratitude to the Spinal Cord Injured Treatment Centre Society and Louise Miller, whose generous donation of the NeuroEducator/ EMG made this work possible, to $\mathrm{Dr} H$ Meeuwsen, of Texas Woman's University, for his editorial comments and to our subjects who were kind enough to donate their time and effort.

\section{References}

1 Burnham RS et al. Shoulder pain in wheelchair athletes - the role of muscle imbalance. Am J Sports Med 1993; 21: 238-242.

2 Nichols PJR, Norman PA, Ennis JR. Wheelchair user's shoulder. Shoulder pain in patients with spinal cord lesions. Scand J Rehabil Med 1979; 11: 29-32.

3 Bayley JC, Cochran TP, Sledge CB. The weight bearing shoulder: The impingement syndrome in paraplegics. J Bone Joint Surg Am 1987; 69: 676-678.

4 Hoeberigs JH, Verstappen FTJ. Muscle soreness in wheelchair basketballers. Int J Sports Med 1984; 5 (suppl): 177-179.

5 Martinez SF: Medical concerns among wheelchair road racers. Phys Sport Med 1989; 17: 63-68.

6 Burnham RS, Curtis KA, Reid DC. Shoulder problems in the wheelchair athlete. In: Pettrone FA (ed). Athletic Injuries of the Shoulder. McGraw Hill: New York, 1995, pp 375-381.

7 Solem-Bertroft E, Thomas K, Westerberg C. The influence of scapular retraction and protection on the width of the subacromical space - an MRI study. Clin Orthop 1993; 252: 99-103.

8 Kibler WB, Chandler TJ. Functional scapular instability in throwing athletes. Presented at the American Orthopaedic Society for Sports Medicine's 15th Annual Meeting, Traverse City, MI, June 19-22, 1989.

9 Kamkar A, Irrgang JJ, Whitney SL. Nonoperative management of secondary shoulder impingement syndrome. J Orthop Sport Phys Ther 1993; 17: 212-223.

10 Wilson PE, Washington RL. Pediatric wheelchair athletics: sport injuries and prevention. Paraplegia 1993; 31: 330-337.

11 Basmajian JV, Blumenstein R. Electrode Placement in EMG Biofeedback. Williams and Wilkins: Baltimore, MD, 1980.

12 Delagi EF, Perotto A. Anatomic Guide for the Electromyographer. 2nd edn. Charles C Thomas: Springfield Il, 1980.

13 Bradley JP, Tibone JE. Electromyographical analysis of muscle action about the shoulder. Clin Sports Med 1991; 10: 789-805.

14 Moseley JB et al. EMG analysis of the scapular muscles during a shoulder rehabilitation program. Am J Sports Med 1992; 20: $128-134$.

15 Daniels L, Worthington C: Muscle Testing. 4th edn. WB Saunders: Philadelphia, 1980.

16 Stein RB, Bruker BS, Ayyar DR. Motor units in incomplete spinal cord injury: electrical activity, contractile properties and the efects of biofeedback. J Neurol Neurosurg Psychiatry 1990; 53: $880-5$

17 Gilai AN. Analysis of turns and amplitude in EMG. In: Computer-Aided Electromyography and Expert Systems. Desmedt JE (ed). Elsevier Science Publishers Biomedical Division: New York, 1989.

18 Ferrarra MS, Davis RW. Injuries to elite wheelchair athletes. Paraplegia 1990; 28: 335-341.

19 Veiersted KB. The reproducibility of test contractions for calibration of electromyographic measurements. Eur J Appl Physiol 1990; 62: 91-98.

20 Laskin et al. Electrical stimulation-assisted rowing exercise in spinal cord injured people. A pilot study. Paraplegia 1993; 31: 534-541.

21 Hagerman FC, Lawrence RA, Mansfield MC. A comparison of energy expenditure during rowing and cycling ergometry. Med Sci Sports Exerc 1988; 20: 479-488. 